\title{
The Witt Ring of a Smooth Projective Curve over a
}

\author{
Finite Field
}

Funk, Jeanne M. Hoobler, Raymond T.

\begin{abstract}
In this paper we calculate the Witt ring $W(C)$ of a smooth geometrically connected projective curve $C$ over a finite field with characteristic other than 2 . We view $W(C)$ as a subring of $W(k(C))$ where $k(C)$ is the function field of $C$. The calculation is then completed using classical results for bilinear spaces over fields.
\end{abstract}

Let $k$ be a finite field of characteristic $q \neq 2$. We will show that the Brauer group of a curve $C$ over $k$ vanishes. The vanishing of the Witt invariant then allows us to represent any symmetric space in $W(C)$ by a form of rank one or two and allows us to write out the multiplication and addition table for $W(C)$ and then recognize it as a quotient of $W(k)\left[{ }_{2} P i c(C)\right]$.

For bilinear spaces over fields, we adopt the notation of (Lam, 2005), in which $<t>$ denotes the rank 1 space with fixed generator $e$ and whose form takes $(e, e)$ to $t \in k$ and $<t_{1}, \ldots, t_{n}>$ is the orthogonal sum $<t_{1}>\perp \cdots \perp<t_{n}>$.

The Witt ring $W(k)$ of $k$ is a four element ring. It consists of 0 , two rank one represented elements $<1>$ (the multiplicative identity) and $\langle s\rangle$, and a nontrivial even rank element. 
The nontrivial even rank element is $\langle 1, s\rangle$ when $q=1(\bmod 4)$ and $\langle 1,1\rangle$ when $q=3(\bmod 4)$.

$W(k)$ has a few properties which are useful for calculational purposes.

- $<1,1>=<s, s>$ for all nondyadic finite fields.

- $<1,1,1>=<1>=<-1>$ when $q=1(\bmod 4)$.

- $<1,1,1>=<s>=<-1>$ when $q=3(\bmod 4)$.

- $<1,1,1,1>=0$ for all nondyadic finite fields.

Let $C$ be a smooth geometrically connected projective curve over $k$ with generic point $\eta$. Assume also that $C$ contains a $k$-rational point.

The natural map $W(C) \rightarrow W(k(C)): E \rightarrow E_{\eta}$ is injective (Balmer and Walter, 2002) and embeds the Witt ring of $C$ into the Witt ring of its function field. This suggests the following notation for spaces represented by orthogonal sums of rank 1 spaces.

$<\mathscr{L}_{\xi}>$ will denote the Witt class represented by a form which maps to $<\xi>\in W(k(C))$ whose underlying vector bundle is the line bundle $\mathscr{L} .<\mathscr{L}_{1, \xi_{1}}, \ldots, \mathscr{L}_{n, \xi_{n}}>$ will denote the orthogonal sum $<\mathscr{L}_{1, \xi_{1}}>\perp \ldots \perp<\mathscr{L}_{n, \xi_{n}}>$.

Due to the rational point, the ring map $W(k) \hookrightarrow W(C)$ induced by the structure map $C \rightarrow \operatorname{Spec}(k)$ is also injective. This map identifies $\langle 1\rangle$ and $\langle s\rangle$ with the Witt classes represented by forms on the structure sheaf.

The following two propositions show that there are $2 n$ distinct Witt classes represented by rank 1 elements where $n$ is the cardinality of the order 2 Picard group ${ }_{2} P i c(C)$. 
Proposition 1. Given $\mathscr{L}, \mathscr{M} \in{ }_{2} \operatorname{Pic}(C)$, if $<\mathscr{L}_{\xi}>=<\mathscr{M}_{\zeta}>\in W(C)$ then $\mathscr{L}=\mathscr{M} \in$ ${ }_{2} \operatorname{Pic}(C)$ and $<\xi>=<\zeta>\in W(k(C))$.

Proof. That $\langle\xi\rangle=\langle\zeta>\in W(k(C))$ is trivial.

Let $\mathscr{L}, \mathscr{M} \in{ }_{2} \operatorname{Pic}(C)$ and $<\mathscr{L}_{\xi}>=<\mathscr{M}_{\zeta}>\in W(C)$. Then $<\mathscr{L}_{\xi}>\perp M=<\mathscr{M}_{\zeta}>\perp M^{\prime} \in \operatorname{Bil}(C) 1$ where $M$ and $M^{\prime}$ are metabolic spaces of equal $\operatorname{rank} 2 m$.

Taking determinants (Knebusch, 1977) on both sides, we see that $\operatorname{det}\left(<\mathscr{L}_{\xi}>\right) \operatorname{det}(M)=\operatorname{det}\left(<\mathscr{M}_{\zeta}>\right) \operatorname{det}\left(M^{\prime}\right),<\mathscr{L}_{\xi}>\left(<-1>^{m}\right)=<\mathscr{M}_{\zeta}>\left(<-1>^{m}\right)$, and $<\mathscr{L}_{\xi}>=<\mathscr{M}_{\zeta}>\in \operatorname{Bil}(C)$.

Proposition 2. Given $\mathscr{L} \in{ }_{2} \operatorname{Pic}(C)$, there are two Witt classes represented by forms whose underlying space is $\mathscr{L}$.

Proof. First, we note that every order 2 line bundle $\mathscr{L}$ is equipped with an isomorphism $\mathscr{L} \otimes \mathscr{L} \rightarrow \mathcal{O}_{C}$, which may be used to define a nondegenerate symmetric bilinear form on $\mathscr{L}$

Two rank 1 forms $(\mathscr{L}, \varphi)$ and $(\mathscr{L}, \psi)$ may differ at most by a global endomorphism of $\mathscr{L}$ as shown in the following diagram:

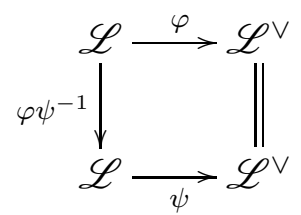

As $\mathscr{E} n d(\mathscr{L}) \cong \mathcal{O}_{C}$, the global endomorphisms of $\mathscr{L}$ are precisely the units of $k$. Thus, $\varphi, \psi$ differ by multiplication $m_{\ell}$ by some unit $\ell \in k$.

\footnotetext{
${ }^{1} B i l(C)$ is the semiring consisting of isomorphism classes of bilinear spaces on $C$
} 
$\varphi$ and $\psi$ represent the same Witt class precisely when $\varphi=m_{\ell} \circ \psi \circ m_{\ell}^{\vee}$ so that $\varphi=\ell^{2} \psi$.

This means that the Witt classes of $C$ associated to $\mathscr{L}$ are in one-to-one correspondence with the square classes of $k$, which correspond to the rank 1 elements of $W(k)$.

We note that multiplication by $<s>\neq<1>\in W(k)$ exchanges the two classes of $W(C)$ represented by forms on $\mathscr{L}$.

Since the function field of $C$ is a $C_{2}$ field we may apply the following classical result for fields (Lam, 2005) Proposition V.3.25.

Proposition 3. Suppose every form of dimension 5 over a field $F$ is isotropic. Then two bilinear spaces $E, E^{\prime}$ are isometric iff $r k(E)=r k\left(E^{\prime}\right) \in \mathbb{Z}, d_{ \pm}(E)=d_{ \pm}\left(E^{\prime}\right) \in W(F)$ and $c(E)=c\left(E^{\prime}\right) \in \operatorname{Br}(F)$ where $d_{ \pm}(-)$is the signed discriminant and the Witt invariant $c(-)$ is the class of the Clifford algebra in the Brauer group $\operatorname{Br}(-)$.

The signed discriminant of $E$ is the rank 1 form $(-1)^{\frac{n(n+1)}{2}} \bigwedge^{n} E$ where $n$ is the rank of $E$. Note that the signed discriminant of a form $<a_{1}, \ldots, a_{n}>$ is $(-1)^{\frac{n(n+1)}{2}}<a_{1} \cdots a_{n}>$.

Details regarding Clifford algebras for forms over fields may be found in (Lam, 2005) Chapter V. A similar definition is used for bilinear spaces on schemes. In the current situation it suffices to know the following:

- $c(E) \in B r(C)$.

- The natural map $\operatorname{Br}(C) \rightarrow \operatorname{Br}(k(C)): A \mapsto A_{\eta}$ is injective.

- $c(E)_{\eta}=c\left(E_{\eta}\right)$.

The following theorem now shows that every bilinear space over $C$ has trivial Witt invariant over $C$ and $k(C)$. 
Theorem 1. $\operatorname{Br}(C)=0$

Proof. We calculate the cohomological Brauer group $H^{2}\left(C_{e t}, \mathbb{G}_{m}\right)$.

There is a spectral sequence $H^{p}\left(\bar{G}, H^{q}\left(\bar{C}, \mathbb{G}_{m}\right)\right) \Rightarrow H^{p+q}\left(C_{e t}, \mathbb{G}_{m}\right)$ (Milne, 1980) III.1.16,2.20 where $\bar{C}$ is the extension of $C$ to the separable closure $k_{\text {sep }}$ of the base field and $\bar{G}=\operatorname{Gal}\left(k_{\text {sep }} / k\right)$. Since $C$ is a curve over a finite field, $\bar{C}$ is also the extension to the algebraic closure.

This is a first quadrant spectral sequence with $E^{2}$ terms as follows:

$E_{0,2}^{2}=H^{0}\left(\bar{G}, H^{2}\left(\bar{C}, \mathbb{G}_{m}\right)\right)$ and $H^{2}\left(\bar{C}, \mathbb{G}_{m}\right)=\operatorname{Br}(\bar{C})=0$ due to the fact that $k(\bar{C})$ is a $C_{1}$ field (Tsen's theorem). Thus, $E_{0,2}^{2}=0$.

$$
\begin{aligned}
E_{2,0}^{2}=H^{2}\left(\bar{G}, H^{0}\left(\bar{C}, \mathbb{G}_{m}\right)\right) & =H^{2}\left(\bar{G}, k_{\text {sep }}^{\times}\right)=\operatorname{Br}(k)=0 \\
E_{1,1}^{2}=H^{1}\left(\bar{G}, H^{1}\left(\bar{C}, \mathbb{G}_{m}\right)\right) & =H^{1}(\bar{G}, \operatorname{Pic}(\bar{C})) . \text { There is a short exact sequence } \\
0 \longrightarrow & \operatorname{Pic}^{0}(\bar{C}) \longrightarrow \operatorname{Pic}(\bar{C}) \stackrel{\text { deg }}{\longrightarrow} \mathbb{Z} \longrightarrow 0
\end{aligned}
$$

Furthermore, $H^{1}\left(\bar{G}, \operatorname{Pic}^{0}(\bar{C})\right)=0$ (Lang's Theorem) and $H^{1}(\bar{G}, \mathbb{Z})=H_{\text {om }}(\bar{G}, \mathbb{Z})=0$ so that $E_{1,1}^{2}=H^{1}(\bar{G}, \operatorname{Pic}(\bar{C}))=0$.

This shows that the cohomological Brauer group, hence the Brauer group, is trivial.

Theorem 1 gives relations $<\mathscr{L}_{\xi}, \mathscr{M}_{\zeta}>=<1, \mathscr{L} \mathscr{M}_{\xi \zeta}>$ in $W(C)$ as well as allowing us to show the following.

Proposition 4. Every class $E \in W(C)$ has a representative of the form $\left\langle\mathscr{L}_{\xi}>\right.$ or $<1,-\mathscr{L}_{\xi}>$ where $<\mathscr{L}_{\xi}>=d_{ \pm}(E)$.

Proof. Consider the image $E_{\eta}$ of $E$ in the function field. 
$E_{\eta}$ has an anisotropic representative $V$ of rank less than five. We will show that this representative must, in fact, have rank less than three.

We first note that the Witt invariant $c(V)$ is trivial.

The tertiary case now follows from (Lam, 2005) Proposition V.3.22.

It remains to show that a rank four form cannot be anisotropic. We consider a diagonalisation $<a_{1}, a_{2}, a_{3}, a_{4}>$ of $V$. Since $c(V)$ is trivial, Proposition 3 shows that $V$ is isometric to $<1,1,1, a_{1} a_{2} a_{3} a_{4}>=<-1, a_{1} a_{2} a_{3} a_{4}>\in W(k(C))$.

Calculations such as $<1,-\mathscr{L}_{\xi}>\perp<\mathscr{M}_{\zeta}>=<1,1,-\mathscr{L} \mathscr{M}_{\xi \zeta}>=<\mathscr{L} \mathscr{M}_{\xi \zeta}>$ now give us the following tables of arithmetic for $W(C)$.

Multiplication:

\begin{tabular}{c|cc} 
& $<\mathscr{L}_{\xi}>$ & $<1,-\mathscr{L}_{\xi^{\prime}}^{\prime}>$ \\
\hline$<\mathscr{M}_{\zeta}>$ & $<\mathscr{L} \mathscr{M}_{\xi \zeta}>$ & $<1,-\mathscr{L}_{\xi^{\prime}}^{\prime}>$ \\
$<1,-\mathscr{M}_{\zeta^{\prime}}^{\prime}>$ & $<1,-\mathscr{M}_{\zeta^{\prime}}^{\prime}>$ & 0
\end{tabular}

Note, in particular, that $<\mathscr{L}_{\xi}><\mathscr{L}_{\xi}>=<1>$ and $<\mathscr{L}_{\xi}><\mathscr{L}_{s \xi}>=<s>$

Addition:

\begin{tabular}{c|cc} 
& $<\mathscr{L}_{\xi}>$ & $<1,-\mathscr{L}_{\xi^{\prime}}^{\prime}>$ \\
\hline$<\mathscr{M}_{\zeta}>$ & $<1, \mathscr{L}_{\mathscr{M}_{\xi \zeta}>}$ & $<\mathscr{M} \mathscr{L}_{\zeta^{\prime}}^{\prime}>$ \\
$<1,-\mathscr{M}_{\zeta^{\prime}}^{\prime}>$ & $<\mathscr{M}^{\prime} \mathscr{L}_{\zeta^{\prime} \xi}>$ & $<1,-\mathscr{M}^{\prime} \mathscr{L}_{\zeta^{\prime} \xi}>$
\end{tabular}

Note that $\left.\left\langle\mathscr{L}_{\xi}\right\rangle \perp<\mathscr{L}_{\xi}\right\rangle=<1,1>$ and $\left.\left.\left\langle\mathscr{L}_{\xi}\right\rangle \perp<\mathscr{L}_{s \xi}\right\rangle=<1, s\right\rangle$.

In fact, $W(C)$ can be expressed quite nicely as a quotient of the group ring $W(k)\left[{ }_{2} \operatorname{Pic}(C)\right]$. 
Theorem 2. $W(C) \cong W(k)\left[{ }_{2} \operatorname{Pic}(C)\right] / \mathscr{R}$ where $\mathscr{R}$ is generated by the relations of the form

$$
<1>-<u>\mathscr{L}-<v>\mathscr{M}+<u v>\mathscr{L} \mathscr{M}
$$

with $\left\langle u>,<v>\in W(k)\right.$ and $\mathscr{L}, \mathscr{M} \in{ }_{2} \operatorname{Pic}(C)$.

Proof. Fix a form $\left\langle\mathscr{L}_{\xi}>\right.$ on each $\mathscr{L} \in{ }_{2} \operatorname{Pic}(C)$.

Define a map $W(k)\left[{ }_{2} \operatorname{Pic}(C)\right] / \mathscr{R} \rightarrow W(C)$ by sending $<s>\mathscr{L}$ to $<\mathscr{L}_{s \xi}>$ and extending by linearity.

This map is clearly a well defined surjection of commutative rings.

To show injectivity let $f \in W(k)\left[{ }_{2} P i c(C)\right] / \mathscr{R}$ map to a form $E=<\mathscr{L}_{1, u_{1} \xi_{1}}, \ldots, \mathscr{L}_{n, u_{n} \xi_{n}}>=0 \in W(C)$. Using the relations $<1,1>=<-1,-1>$ and $<\mathscr{L}_{u \xi}, \mathscr{M}_{v \zeta}>=<1, \mathscr{L}_{\mathscr{M}_{u v \xi \zeta}}>$ we may rewrite $E$ as $< \pm 1, \mathscr{L}_{1} \cdots \mathscr{L}_{n, u_{1} \cdots u_{n} \xi_{1} \cdots \xi_{n}}>$. Using the corresponding relations $<1>+<1>+<1>+<1>$ and $<1>-<u>\mathscr{L}-<v>\mathscr{M}+<u v>\mathscr{L} \mathscr{M}$ in $W(k)\left[{ }_{2} P i c(C)\right] / \mathscr{R}$, we may write $f$ as $< \pm 1>+<u_{1} \cdots u_{n}>\mathscr{L}_{1} \cdots \mathscr{L}_{n}$. In the +1 case, $E$ and $f$ are trivial precisely when $<u_{1} \cdots u_{n} \mathscr{L}_{1} \cdots \mathscr{L}_{n}>=<1>$. In the -1 case, both $E$ and $f$ are trivial precisely when $<u_{1} \cdots u_{n} \mathscr{L}_{1} \cdots \mathscr{L}_{n}>=<-1>$

\section{References}

P. Balmer and C. Walter. A gersten-witt spectral sequence for regular schemes. Annales Scientifiques de l'École Normale Supérieure, 35(1):127-152, 2002.

Manfred Knebusch. Symmetric bilinear forms over algebraic varieties. In Conference on Quadratic Forms-1976 (Proc. Conf., Queen's Univ., Kingston, Ont., 1976), pages 103- 
283. Queen's Papers in Pure and Appl. Math., No. 46. Queen's Univ., Kingston, Ont., 1977.

T. Y. Lam. Introduction to quadratic forms over fields, volume 67 of Graduate Studies in Mathematics. American Mathematical Society, Providence, RI, 2005. ISBN 0-8218-1095-2.

James S. Milne. Étale cohomology, volume 33 of Princeton Mathematical Series. Princeton University Press, Princeton, N.J., 1980. ISBN 0-691-08238-3. 